\title{
The Right Time and "PI ACE”: Optimal Management of Perioperative Angiotensin-Converting Enzyme Inhibitors
}

\author{
Vineet Chopra, MD, MSc ${ }^{1 *}$, Duminda N. Wijeysundera, MD, PhD ${ }^{2,3}$
}

\begin{abstract}
${ }^{1}$ The Patient Safety Enhancement Program of the Ann Arbor VA Healthcare System and Department of Medicine, University of Michigan Health System, Ann Arbor, Michigan; 'Li Ka Shing Knowledge Institute of St. Michael's Hospital, Toronto, Ontario, Canada; ${ }^{3}$ Department of Anesthesia, Toronto General Hospital and University of Toronto, Toronto, Ontario, Canada.
\end{abstract}

The management of perioperative medications is a tale of progressive scientific enquiry. Although long-term use of agents such as aspirin or statins improves clinical outcomes, use during surgery ranges from problematic to protective. The delicate balance between proven long-term benefits in the nonoperative setting versus short-term uncertainty in the perioperative setting must be assessed using a lens that incorporates patient risk, surgical process, and pharmacodynamic principles. Advances in our understanding of perioperative physiology, coupled with robust clinical and outcome data, have led to new knowledge and insights regarding how best to manage these medications. As well illustrated by the near $180^{\circ}$ change in the use of perioperative $\beta$ blockers to prevent adverse cardiovascular outcomes, these new data have led to substantial progress in surgical safety and patient outcomes.

In this issue of the Journal of Hospital Medicine, 2 retrospective cohort studies add to this growing body of evidence by examining risks associated with use of angiotensin-converting enzyme (ACE) inhibitors in the perioperative setting. In the first study, conducted at a single academic medical center, Nielson and colleagues evaluate the association of preoperative ACEinhibitor use with hypotension and acute kidney injury in patients undergoing major elective orthopedic surgery. ${ }^{1}$ The authors report that patients receiving ACE-inhibitors were not only more likely to experience hypotension after induction of anesthesia $(12.2 \%$ vs $6.7 \%, P=0.005)$, but also were more likely to develop postoperative acute kidney injury (odds ratio [OR]: $2.68,95 \%$ confidence interval [CI]: $1.25-1.99)$. In the second study which focused solely on patients who were receiving preoperative ACEinhibitor therapy, Mudumbai and colleagues used a national Veterans Affairs database to examine the association between failure to resume ACE-inhibitor treatment after surgery and outcomes at 30 days. $^{2}$

*Address for correspondence and reprint requests: Vineet Chopra, MD, North Campus Research Complex, 2800 Plymouth Road, Building 16 Room 432W, Ann Arbor, Ml 48109; Telephone: 734-936-5216; Fax: 734689-4420; E-mail: vineetc@umich.edu

Additional Supporting Information may be found in the online version of this article.

Received: March 27, 2014; Accepted: March 28, 2014 2014 Society of Hospital Medicine DOI 10.1002/jhm.2203

Published online in Wiley Online Library (Wileyonlinelibrary.com).
The authors found that failure to resume treatment 14 days after surgery was not only common (affecting 1 in 4 patients), but was also associated with increased 30-day mortality (hazard ratio: 3.44, 95\% CI: 3.303.60). ${ }^{2}$ Taken together, these 2 studies shed new light on clinical practice and policy implications for the use of these agents in the surgical period. Both sets of authors should be congratulated on moving the needle forward in this enquiry.

ACE-inhibitors physiologically mediate their effects by preventing the formation of the potent vasoconstrictor angiotensin-II from its precursor angiotensin-I. In doing so, they decrease arterial resistance, increase venous capacitance, decrease glomerular filtration pressure, and promote natriuresis. These vascular effects have key benefits for the management of a number of chronic diseases including hypertension, congestive heart failure, and diabetic nephropathy. However, these clinical alterations are often problematic in the perioperative setting. For example, ACEinhibitors may cause vasoplegia during anesthetic administration, commonly manifested as hypotension during induction. ${ }^{3}$ This hemodynamic alteration has been viewed as being so precarious, that some authors recommend withholding ACE-inhibitors prior to major cardiovascular procedures such as coronary artery bypass grafting. ${ }^{4}$ Although hypotension leads to management challenges (often increasing vasoconstrictor requirements), several studies report that ACEinhibitor use during surgery may also be associated with increased risks of acute kidney injury and mortality. ${ }^{5,6}$ However, despite these data, existing literature has not shown a consistent association between ACE-inhibitor use and adverse postoperative outcomes. For example, a propensity-matched cohort study of 79,228 patients at the Cleveland Clinic found no difference in hemodynamic characteristics, vasopressor requirements, or cardiorespiratory complications among patients who were or were not using ACE-inhibitors during noncardiac surgery. ${ }^{7}$ Furthermore, some research has also found that withdrawal of ACE-inhibitors may itself lead to harm. For instance, a contemporary study reported that withdrawal of ACE-inhibitor therapy in patients undergoing coronary artery bypass was associated with increased in-hospital cardiovascular events. ${ }^{8}$ Given this uncertainty, it is not surprising that management 
of ACE-inhibitors in the perioperative period remains a subject of ongoing controversy with clinical reviews often recommending consideration of the risks and benefits associated with use of these agents. ${ }^{9}$

It is important to note that driver of this ambiguity is the very design of relevant studies. For instance, studies that focus on patients undergoing coronary artery bypass grafting surgery have limited external validity, as these patients are very different from those who undergo elective hip or knee replacement (such as those included by Nielsen and colleagues). Additionally, many studies suffer from methodological constraints or biases. For instance, retrospective observational studies often suffer from selection bias and residual confounding; that is, the individuals chosen for inclusion in the study and the variables available for analysis are often limited by available data, curtailing the conclusions that can be generated. Although the use of multivariable regression or propensity score techniques helps address these limitations, residual confounding by unmeasured variables always remains a threat to statistical inference. The potential influence for this bias is particularly relevant when examining the results of the study by Nielsen and colleagues. Another important limitation is survivor bias, a problem inherent in the study by Mudumbai and colleagues. Put simply, for a patient to resume ACE-inhibitors after surgery, this same patient, must also survive for at least 15 to 30 days after surgery. Thus, for some patients, failure to resume this treatment may simply be a marker of early mortality rather than failure to resume the ACE itself. The potential influence of this bias is supported by an included sensitivity analysis, where a large change in the adjusted OR was observed when patients suffering early mortality were excluded. This swing in effect size suggests that biases related to comparing patients who survived to those who did not with respect to ACE use may, in part, account for the results of the study.

These limitations aside, the studies brought forth by both authors help inform practice with respect to the use of these agents during surgery. In this context, 3 paradigms are relevant for practicing hospitalists.

First, if a patient is maintained on an ACE-inhibitor before surgery, should the medication be temporarily held before surgery to minimize hypotension during anesthesia? The study by Nielsen and colleagues (comparing those on ACE-inhibitor treatment to those without), in addition to the evidence generated from other studies in this area ${ }^{10-12}$ suggest that this is a rational decision. Although the existence of a "withdrawal" state from abrupt cessation of ACEinhibitor use is theoretically plausible, this has yet to be reliably reported in the literature. Given the short half-life of most ACE-inhibitors, cessation 24 hours before surgery appears to be the most pragmatic clinical approach.

Second, if a patient is on an ACE-inhibitor before surgery, when should the medication be resumed after surgery? The findings from the study by Mudumbai and colleagues, in addition to contemporary evidence, ${ }^{7,8,13}$ support the resumption of these agents as soon as possible following operative intervention. Once hemodynamic stability and volume status have been assured, risks associated with postoperative ACE-inhibitor use appear to be outweighed by benefits, though specific care is likely necessary in those with preexisting renal dysfunction. ${ }^{14}$ A program that ensures reconciliation of medications in the postoperative setting may be valuable in ensuring that such treatment is restarted.

Third, if a patient is not on ACE-inhibitor therapy, should this be started before surgery for perioperative or long-term benefit? Although neither study examines this issue, the potential for significant risk make this an unattractive option. Future interventional studies with thoughtfully weighed safety parameters may be necessary to assess whether such a paradigm may be valuable.

The studies included in this issue of the Journal of Hospital Medicine suggest that the use of ACE-inhibitors during the perioperative period may be considered a function of time and place. Resuming ACEinhibitors and cessation of treatment at specific intervals in relation to surgery can help ensure positive outcomes. Hospitalists have an important role in this regard, as they are ideally situated to manage these agents in the many patients undergoing surgery across the United States.

Disclosures: Dr. Wijeysundera is supported by a Clinician-Scientist Award from the Canadian Institutes of Health Research, and a Merit Award from the Department of Anesthesia at the University of Toronto. Dr. Chopra is supported by a Career-Development Award (1K08HS022835-01) from the Agency of Healthcare Research and Quality.

\section{References}

1. Nielson E, Hennrikus E, Lehman E, Mets B. Angiotensin axis blockade, hypotension, and acute kidney injury in elective major orthopedic surgery. J Hosp Med. 2014;9(5):283-288.

2. Mudumbai SC, Takemoto S, Cason BA, Au S, Upadhyay A, Wallace AW. Thirty-day mortality risk associated with postoperative nonresumption of angiotensin-converting enzyme inhibitors: a retrospective study of the Veterans Affairs Healthcare System. J Hosp Med. 2014; 9(5):289-296.

3. Rosenman DJ, McDonald FS, Ebbert JO, Erwin PJ, LaBella M, Montori VM. Clinical consequences of withholding versus administering renin-angiotensin-aldosterone system antagonists in the preoperative period. J Hosp Med. 2008;3(4):319-325.

4. Tuman KJ, McCarthy RJ, O'Connor CJ, Holm WE, Ivankovich AD. Angiotensin-converting enzyme inhibitors increase vasoconstrictor requirements after cardiopulmonary bypass. Anesth Analg. 1995; 80(3):473-479.

5. Miceli A, Capoun R, Fino C, et al. Effects of angiotensin-converting enzyme inhibitor therapy on clinical outcome in patients undergoing coronary artery bypass grafting. I Am Coll Cardiol. 2009;54(19): 1778-1784.

6. Railton CJ, Wolpin J, Lam-McCulloch J, Belo SE. Renin-angiotensin blockade is associated with increased mortality after vascular surgery. Can J Anaesth. 2010;57(8):736-744.

7. Turan A, You J, Shiba A, Kurz A, Saager L, Sessler DI. Angiotensin converting enzyme inhibitors are not associated with respiratory complications or mortality after noncardiac surgery. Anesth Analg. 2012; 114(3):552-560.

8. Drenger B, Fontes ML, Miao Y, et al. Patterns of use of perioperative angiotensin-converting enzyme inhibitors in coronary artery bypass graft surgery with cardiopulmonary bypass: effects on in-hospital morbidity and mortality. Circulation. 2012;126(3):261-269. 
9. Auron M, Harte B, Kumar A, Michota F. Renin-angiotensin system antagonists in the perioperative setting: clinical consequences and recommendations for practice. Postgrad Med J. 2011;87(1029):472-481.

10. Coca SG, Garg AX, Swaminathan M, et al; TRIBE-AKI Consortium. Preoperative angiotensin-converting enzyme inhibitors and angiotensin receptor blocker use and acute kidney injury in patients undergoing cardiac surgery. Nephrol Dial Transplant. 2013;28(11): $2787-2799$.

11. Yoo YC, Youn YN, Shim JK, Kim JC, Kim NY, Kwak YL. Effects of renin-angiotensin system inhibitors on the occurrence of acute kidney injury following off-pump coronary artery bypass grafting. Circulation J. 2010;74(9):1852-1858.
12. Hasija S, Makhija N, Choudhury M, Hote M, Chauhan S, Kiran U. Prophylactic vasopressin in patients receiving the angiotensin-converting enzyme inhibitor ramipril undergoing coronary artery bypass graft surgery. J Cardiothorac Vasc Anesth. 2010;24(2):230-238.

13. Hanninen M, McAlister FA, Bakal JA, van Diepen S, Ezekowitz JA. Neither diabetes nor glucose-lowering drugs are associated with mortality after noncardiac surgery in patients with coronary artery disease or heart failure. Can J Cardiol. 2013;29(4):423-428.

14. Zacharias M, Conlon NP, Herbison GP, Sivalingam P, Walker RJ, Hovhannisyan K. Interventions for protecting renal function in the perioperative period. Cochrane Database Syst Rev. 2008;(4): CD003590. 